\title{
High-Resolution Measurements of Middle Ear Gas Volume Changes in the Rabbit Enables Estimation of its Mucosal $\mathrm{CO}_{2}$ Conductance
}

\author{
Yael Marcusohn, ${ }^{1}$ Joris J. J. Dirckx, ${ }^{1}$ and Amos $\mathrm{Ar}^{2}$ \\ ${ }^{1}$ Laboratory of Biomedical Physics, University of Antwerp, Antwerp B-2020, Belgium \\ ${ }^{2}$ Department of Zoology, Faculty of Life Sciences, Tel Aviv University, Ramat Aviv, Tel Aviv 69978, Israel
}

Received: 23 September 2005; Accepted: 2 April 2006; Online publication: 25 May 2006

\begin{abstract}
Transmucosal $\mathrm{CO}_{2}$ exchange in the middle ear (ME) of the New Zealand White rabbit (Oryctolagus cuniculus) was studied using an accurate novel detecting and recording system for measuring gas volume changes at constant pressure, based on a principle that was previously used by Kania et al. (Acta Otolaryngol 124:408-410, 2004). After the ME cavity was washed with ambient air, the initial diffusion rate of $\mathrm{CO}_{2}\left(\dot{V}_{\mathrm{i}} \mathrm{CO}_{2}\right)$ from the blood perfusing the $\mathrm{ME}$ mucosa was calculated from gas volume change measurements. In nine cases, the $\dot{V}_{\mathrm{i}} \mathrm{CO}_{2}$ calculated after normalization due to shifts in baseline was $314 \pm$ $112 \mu \mathrm{L} \cdot \mathrm{h}^{-1}$ (mean $\pm \mathrm{SD}$ ). In two cases where normalization was not needed, $\dot{V}_{\mathrm{i}} \mathrm{CO}_{2}$ was $409 \mu \mathrm{L} \cdot \mathrm{h}^{-1}$ (276 and $543 \mu \mathrm{L} \cdot \mathrm{h}^{-1}$ ). Normalization of $\dot{V}_{\mathrm{i}} \mathrm{CO}_{2}$ data was also made in five additional cases where secretion of fluids from the lining of the ear canal was observed. In these cases $\dot{V}_{\mathrm{i}} \mathrm{CO}_{2}$ was $245 \pm 142 \mu \mathrm{L} \cdot \mathrm{h}^{-1}$. No differences were found between results obtained in the three groups. Thus, an overall mean value of $\dot{V}_{\mathrm{i}} \mathrm{CO}_{2}$ of $305 \pm 131 \mu \mathrm{L} \cdot \mathrm{h}^{-1}(n=16)$ was calculated. An effective coefficient of conductance of $\mathrm{CO}_{2}\left(\mathrm{GCO}_{2}\right)$ between the mucosal circulation and the $\mathrm{ME}$ gas cavity of the New Zealand White rabbit was estimated to be $\approx 0.05 \mu \mathrm{L}(\mathrm{h} \cdot \mathrm{Pa})^{-1}$ and compared to the $\mathrm{GCO}_{2}$ estimated for humans in a different study.

Keywords: rabbit, middle ear, gas exchange, $\mathrm{CO}_{2}$ conductance
\end{abstract}

Correspondence to: Yael Marcusohn - Laboratory of Biomedical Physics · University of Antwerp · Antwerp B-2020, Belgium. email: Yael.Marcusohn@ua.ac.be
Abbreviations: $A$-the surface area of a diffusion barrier; $B$-the value of $\Delta V_{t}$ when $t$ approaches infinity; $D_{\mathrm{g}}$-the diffusion coefficient of a gas in a diffusion barrier; EC-ear canal; ET-Eustachian tube; $\mathrm{GCO}_{2}$ - the coefficient of conductance of $\mathrm{CO}_{2}$; i.m. - intramuscular; i.v. - intravenous; $k$-the rate constant of gas volume increase; $L$-thickness of a diffusion barrier; $\mathrm{ME}$ - middle ear; $\mathrm{PaCO}_{2}$ - the partial pressure of $\mathrm{CO}_{2}$ in the arterial blood; $t$-elapsed time; TM-tympanic membrane; $\dot{V}_{g}$-the rate per unit of time of gas diffusion through a barrier; $\dot{V}_{\mathrm{i}} \mathrm{CO}_{2}-$ the initial diffusion rate of $\mathrm{CO}_{2} ; \alpha_{\mathrm{g}}$ - the solubility coefficient of a gas in a diffusion barrier; $\Delta P_{\mathrm{g}}-$ the partial pressure difference of a given gas across a diffusion barrier; $\Delta V_{t}$-the deviation of ME volume with time.

\section{INTRODUCTION}

The middle ear (ME) cleft, a rigid gas-filled cavity, differs in size and morphological structure between species. Whereas some (e.g., humans, guinea pigs, pigs) have complex gas-filled systems containing multiple connected cells (e.g., the mastoid gas cell system in humans) that are connected to the ME cavity itself, others (e.g., rats, rabbits) have a rather simple structure (bulla) (Hellström et al. 1982; Judkins and Li 1997; Goksu et al. 1992; Pracy et al. 1998; Hamada et al. 2002).

Exchange of ME gases $\left(\mathrm{N}_{2}, \mathrm{O}_{2}, \mathrm{CO}_{2}, \mathrm{H}_{2} \mathrm{O}, \mathrm{Ar}\right)$ between the ME and the blood vessels of the mucosa, through the mucosal lining, has been studied in different animal models, usually by measuring $\mathrm{ME}$ pressure variations following disturbances in normal ME gas composition. Such studies include replace- 
ment of the ME gas contents with other gas mixtures. In this way, the normal steady-state condition of ME gas is challenged, and until steady state is reestablished, the general gas exchange process over time can be studied. This general method has been used in different animals, e.g., rabbits (Hamada et al. 2002), rats (Kania et al. 2004), and monkeys (Doyle and Seroky 1994; Doyle et al. 1995, 1999), and also in humans (Aoki et al. 1998; Uchimizu et al. 2005). It has also been described by mathematical models (e.g., Doyle and Alper 1999; Fink et al. 2003).

In the present study, the course of ME gas volume change with time until steady state is reestablished and beyond was followed, after flushing with ambient air the MEs of anesthetized rabbits [with closed inactive Eustachian tubes (ETs)]. An initial increase of the measured ME gas volume at constant ambient pressure that was observed (phase "a"), gradually turned into a linear decrease in volume (phase "b") (Figs. 1 and 4).

\section{Phase "a"}

We assumed that phase "a" was governed by fast diffusion of $\mathrm{CO}_{2}$ from the blood that perfuses the $\mathrm{ME}$ mucosa into the ME, down its partial pressure difference. It was further assumed that due to the air flush of the ME at the beginning of each experiment, the initial concentration of $\mathrm{CO}_{2}$ in the ME was very close to zero. Thus, the value of the initial partial pressure difference of $\mathrm{CO}_{2}$ between the blood and the ME was assumed to be equal to the partial pressure of $\mathrm{CO}_{2}$ in the blood.

The assumption that the initial increase in volume (Kania et al. 2004; this study) or in pressure (Hamada et al. 2002; Aoki et al. 1998; Uchimizu et al. 2005) can be attributed to an initially relatively fast $\mathrm{CO}_{2}$ diffusion into the ME space from the blood circulating in the mucosa is based on the fact that its mucosal solubility is much higher than that of the other gases involved (Sadé and Ar 1997; Doyle and Seroky 1994; Doyle et al. 1999; Fink et al. 2003). This assumption was further supported experimentally in rabbits where phase "a" did not occur when the ME was initially flushed with a gas mixture containing $5 \% \quad \mathrm{CO}_{2}$ (similar to venous blood; Hamada et al. 2002).

\section{Phase "b"}

Because the rate of $\mathrm{N}_{2}$ exchange is slower than that of $\mathrm{O}_{2}$ and much slower than that of $\mathrm{CO}_{2}$ (Doyle and Seroky 1994; Doyle et al. 1995, 1999), while $\mathrm{O}_{2}$ and $\mathrm{CO}_{2}$ reach a steady-state situation in which they are nearly in equilibrium with the surrounding tissues and blood relatively quickly, the difference for $\mathrm{N}_{2}$ is maintained as long as the total ME gas pressure is maintained. This is the situation under normal con- ditions when the ET function admits gas from the nasopharynx (Sadé and Ar 1997).

It was assumed that if the ET is closed, the linear decrease in volume with time that was measured in phase "b" represented a steady-state condition, gov-
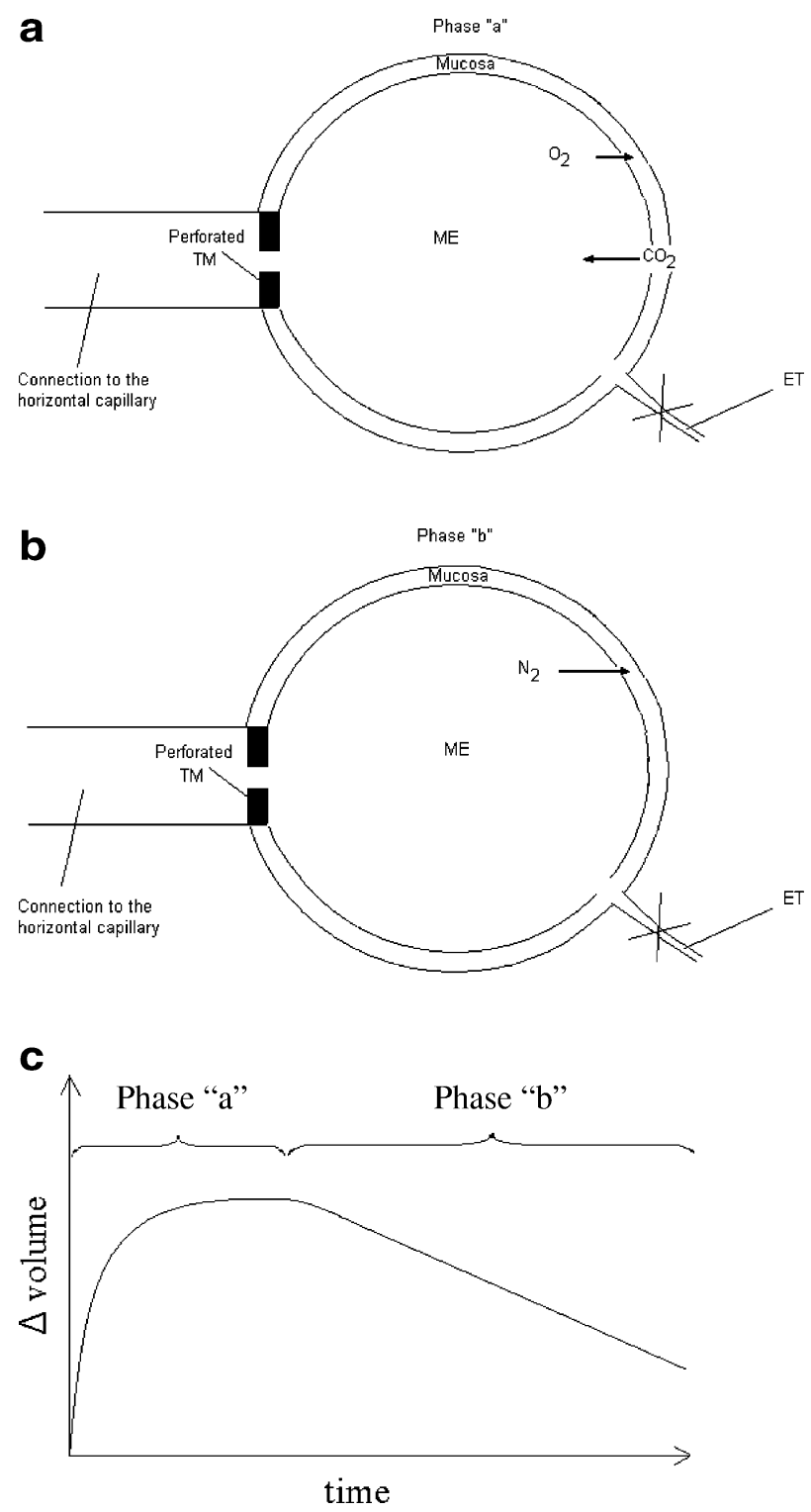

FIG. 1. (a) Phase "a": The volume change is governed by diffusion of $\mathrm{CO}_{2}$ from the blood that perfuses the ME mucosa into the airflushed ME, down its partial pressure difference. The influence of $\mathrm{O}_{2}$ that diffuses down its partial pressure difference from the ME into the blood is assumed to manifest itself at a later part of that phase [when the curve presented in (c) approaches a plateau; the partial pressure of $\mathrm{H}_{2} \mathrm{O}$ does not change and is not shown in the scheme. The overall volume change in phase "a" is depicted in (c)]. (b) Phase "b": The volume change is governed by diffusion of $\mathrm{N}_{2}$ from the ME into the blood that perfuses the ME mucosa, down its partial pressure difference (following an equilibration of $\mathrm{O}_{2}$ and $\mathrm{CO}_{2}$ with the mucosal blood circulation). There is no change in the partial pressure of water vapor (not shown in scheme). (c) A simplified theoretical illustration of the expected total gas volume changes in the ME (phases "a" and "b") at a constant total ME pressure. 
erned by net continuous $\mathrm{N}_{2}$ diffusion from the ME into the blood followed by reequilibration of the partial pressures of $\mathrm{O}_{2}$ and $\mathrm{CO}_{2}$ down their partial pressure differences but without replenishing the gas through the ET.

In our experimental conditions, where the animals are anesthetized, the ET is not functioning; thus, the compensation for absence of ventilation is the volume reduction of the experimental system employed (see "Materials and methods"). It is depicted as a linear decrease in ME volume (Ar et al. 2003; Kania et al. 2004; Fig. 4).

Measuring ME gas exchange on the basis of pressure change at a constant volume has essential drawbacks: The results are highly susceptible to even the smallest leaks as the exchange processes involve relatively little gas exchange. Leakage will underestimate the pressure increase in phase "a" and the following pressure decrease will erroneously be interpreted as a volume decrease. In addition, the total pressure changes would, by nature, change the partial pressures of gases involved, thus changing the gas pressure differences between the ME gas and the gas in the ME circulation. In contrast to pressure measurements (=constant volume), the volume change measurements (=constant pressure) are independent of the system volume (including its dead space) and present directly and quantitatively the gas volume changes involved. Moreover, isopressure measurements are less susceptible to very small leakages than isovolume measurements. In an absolute pressureless volume change measurement, this artifact is excluded; in a near-pressureless measurement like ours, it is very much attenuated.

Various findings indicate that the ME is susceptible to pressure. Based on their measurements of pars flaccida (PF) motions, Hellström and Stenfors (1983) argued that the PF may act as a pressure receptor, a view that was supported by the discovery of nerve endings in the tympanic membrane (TM) (e.g., Wilson 1911; Nagai and Tono 1989; Nagai 1995). ME pressure is continuously regulated during the day (Tideholm et al. 1998; Dirckx et al. 2001) by action of the ET muscles, possibly through neural feedback loops (Eden and Gannon 1987; Eden et al. 1990). In a very recent work (Nielsen et al. 2005), actual brain activity triggered by changing ME pressure has been demonstrated. Although there is no conclusive evidence yet, all these findings indicate that ME pressure is actively regulated.

Ar et al. (2003) measured ME gas volume changes at constant pressure equal to ambient pressure. In the system they used, the displacement of a droplet in a horizontal air-filled capillary (see "Materials and methods") was measured manually using successive image frames taken by a video camera.
The aim of the present study was to describe transmucosal $\mathrm{CO}_{2}$ exchange and thereby to evaluate experimentally the coefficient of conductance of $\mathrm{CO}_{2}$ in the $\mathrm{ME}$ of the rabbit, using a high-resolution measurement system that was developed in our laboratory.

The importance of the isopressure method in the clinical setting was emphasized before by Sadé et al. (2005) in the context of hyperectasis. The method has a general value as well, as it can be used to calculate conductance of various gases of interest (e.g., $\mathrm{N}_{2} \mathrm{O}$ ), using the same technique that was used in the present study to estimate $\mathrm{CO}_{2}$ flux and conductance. It may also be used to validate theoretical estimations (Fink et al. 2003; Kanick et al. 2005) of that variable in different gases.

\section{MATERIALS AND METHODS}

The procedure used in the present study included an initial flushing of the ME with ambient air in ears with preperforated TMs that were exposed to the atmosphere. The gas composition of such MEs that were exposed to the atmosphere (through a ventilating tube or a chronic perforation) was found to be inbetween that of air and normal ME gas composition (Felding 1998). Thus, in the present study, a flush of the ME with ambient air allowed us to start the measurements with a well-defined ME gas composition to avoid the need for use of additional sensors and, at the same time, to study the exchange process of a single gas. The same system can thus be used in the future for similar studies using other gas mixtures for the initial flush of the ME.

\section{Animals}

The animal model selected for the present study was the rabbit. Compared with other available laboratory animals, it has a relatively large ME volume of approximately $300 \mu \mathrm{L}$ (that of the rat is one order of magnitude smaller). It was expected that performing measurements on a ME with a relatively large gas volume would increase the accuracy of the measurements and allow more time to follow the gas volume changes.

Measurements that were made on 16 male New Zealand White rabbits (Oryctolagus cuniculus) (2.8 \pm $0.3 \mathrm{~kg}$ ) are presented. The rabbits were maintained under artificial light cycle conditions (8L:16D). Food and water were supplied ad lib.

The rabbits were anesthetized by intramuscular injections of ketamine (35 mg/kg; Anesketin, 100 $\mathrm{mg} / \mathrm{mL}$, Eurovet Animal Health, Belgium/the Netherlands) and xylazine (5 mg/kg; Rompun $2 \%$, Bayer, 
Belgium). During the experiment, anesthesia was maintained by continuous intravenous infusion of a mix of anesthetics at a constant rate (ketamine, $0.221 \mathrm{~mL}$ $\mathrm{kg}^{-1} \mathrm{~h}^{-1}$; xylazine, $0.111 \mathrm{~mL} \mathrm{~kg}^{-1} \mathrm{~h}^{-1}$; Ringer, $5 \mathrm{~mL}$ $\mathrm{kg}^{-1} \mathrm{~h}^{-1}$ ), using an infusion pump. Ophthalmic ointment (Lacrytube, Vitaris, Belgium) was applied to the eyes to prevent drying of the cornea during the experiments.

Body temperature was maintained at $38.9 \pm 0.8^{\circ} \mathrm{C}$ by using a thermoregulated heating blanket (Harvard Apparatus, USA). Pulse and blood $\mathrm{O}_{2}$ saturation were monitored using a pulse oximeter (Oxypleth, Model 520A, Novametrix, USA; heart rate $=191 \pm 18$ beats $/$ min; blood hemoglobin $\mathrm{O}_{2}$ saturation $=87 \pm 5 \%$ )

The study was performed according to the regulations of the local ethical committee.

The TM was examined by otomicroscopy at the beginning of each experiment to verify that the ME and the TM were healthy. The TM was perforated under general anesthesia, using the surgical microscope.

The ear canal of the rabbit is very complex in shape. Behind the distal soft part of the cartilaginous ear canal, the proximal cartilaginous part is curved by an angle of nearly $90^{\circ}$. This part ends on the bony orifice of the ME, and the annulus of the TM is positioned nearly at a right angle to that orifice. A mold of the ear canal is shown in Fig. 2. Both the deep part of the soft cartilaginous ear canal and the bony part have a very delicate epidermis that is sensitive to touch. The dermis under it becomes edematous and bleeds easily. By stretching the canal as much as possible, one can just obtain a very oblique view of the TM. During the experiments, it became

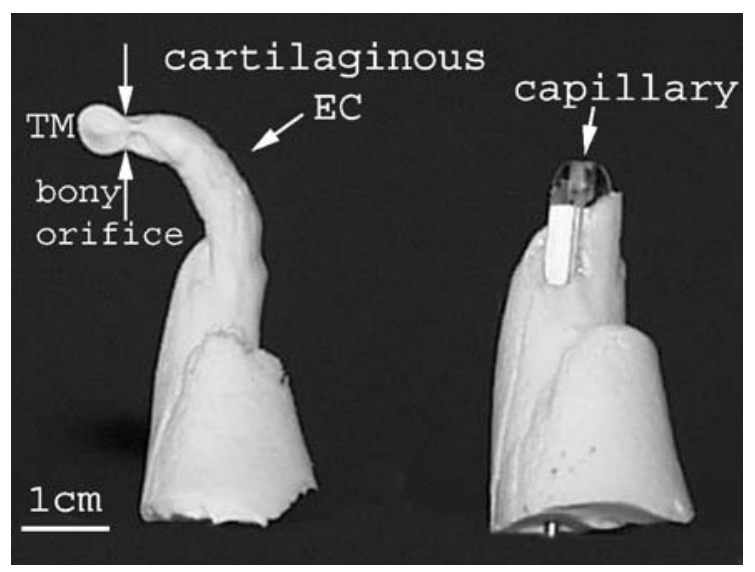

FIG. 2. Left: a complete impression of a rabbit ear canal (EC) up to the tympanic membrane (TM) level. A $90^{\circ}$ curvature followed by a small orifice makes the TM difficult to reach. Right: connection of the measurement system to the ME through the ear canal: The impression material forms an airtight fit between the glass capillary (tip is seen) and the ear canal. The capillary does not enter the proximal part of the ear canal in order to prevent damage to the vulnerable lining of that part. clear that blood accumulated frequently in the deep part of the ear canal if the sensitive part of the ear canal was touched during TM perforation. Ten measurements were performed using only a speculum and a small stiff wire for puncturing the TM. Four of them were discarded, either due to excessive bleeding in the ear canal (correlated with a negligible volume change comparing to the other cases), noise in the system (electrical noise that was intense enough to hinder a large part of the measured curve), or when phase "a" was not measured (due to a technical problem in the initial step when the ME was flushed).

Since some of these measurements (three out of six cases) showed continuous volume increases attributable to extremely small amounts of fluid or blood that accumulated in the ear canal, we developed a special tool to perform the perforation. Ten measurements were performed using that tool (in two of them the same continuous volume increase due to fluid or blood accumulation was observed). Briefly, a thin tube was mounted on the shaft of a rigid endoscope (diameter, $2.7 \mathrm{~mm}$; length, $11 \mathrm{~cm}$; Karl Storz, Germany) through which a thin metal wire could be guided under inspection into the ear canal. The endoscope was introduced to a level just before the bend in the ear canal. The wire was manipulated to stay away from the ear canal walls until it reached the TM, where it caused the desired perforation. We prepared wires with a little sphere attached to the end, but even touching with such a blunt object irritates the canal wall. Using the endoscope, however, we managed to perforate the $\mathrm{TM}$ in a few ears without touching the medial part of the ear canal. In these cases, no bleeding or fluid formation was observed.

In the "Results" section we report separately results with and without fluid formation.

A glass capillary (inner diameter, $0.72 \mathrm{~mm}$ ) was mounted horizontally on a custom-made optoelectronic device (described in detail below). One end of the capillary was open to ambient air and the other was connected to the ME through a three-way stopcock (Fig. 3). This stopcock was connected to a second stopcock with a polyethylene tube (Fig. 3), to which a pressure transducer (Fluke 700PD2, USA) and a 1-mL syringe filled with air, were connected. The system was connected to the ME through the first three-way stopcock by a polyethylene tube connected to a short greased glass capillary. The glass capillary was introduced into the ear canal (Figs. 2 and 3) and was kept in place by injecting a fast-setting ear molding material (Dreve-Otoplastik, Germany) around it. After checking that the system was airtight (see below), a water droplet (containing a surfactant, as explained below) was introduced into the capillary. The ME was air-flushed using the 1-mL syringe. 


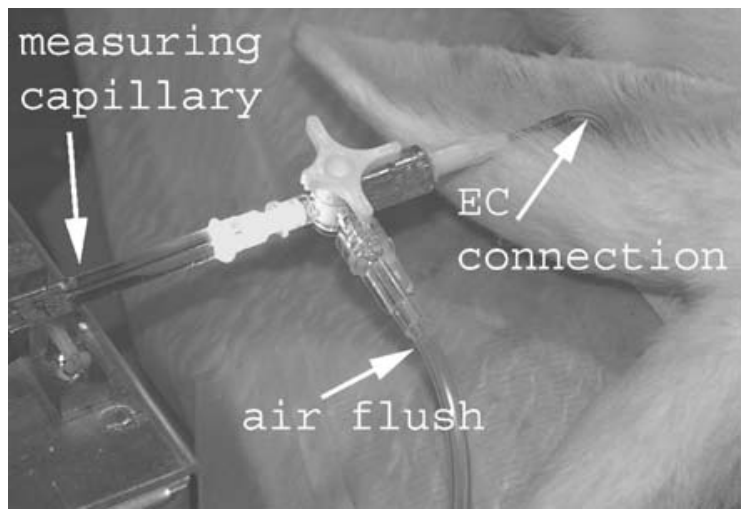

FIG. 3. Connections between the measuring system and the ME: A glass capillary (left) was mounted horizontally on a custom-made optoelectronic device. One end of the capillary was open to ambient air and the other was connected to the ME through a three-way stopcock by a polyethylene tube connected to a short greased glass capillary. The glass capillary was introduced into the ear canal (EC) and was kept in place by injecting a fast-setting ear molding material around it (not shown). Air flush was performed through a second polyethylene tube connected to this stopcock (explained in detail in the "Materials and methods" section).

About $4 \mathrm{kPa}$ of pressure was applied to ensure opening of the ET and thus flushing the entire ME through the ET. The system was then closed and the automatic monitoring of gas volume changes started. At the end of the experiment the ME was examined again under the microscope.

\section{Checking the airtightness of the system}

As the capillary in such experiments is in a horizontal position and the friction is very low, negligible pressure is needed to move the droplet. To minimize the effect of surface tension, we added some surfactant to the droplet fluid. A droplet of about $5 \mathrm{~mm}$ long will start moving down when the capillary is placed in the vertical position, showing that a pressure of less than $50 \mathrm{~Pa}$ is needed to make the droplet move. Hence, it is safe to say that measurements were done at constant pressure and that we recorded pure volume changes. However, the need for up to $\sim 50 \mathrm{~Pa}$ pressure difference to make the droplet move can cause artifacts if the system is not perfectly airtight: when volume increases very slowly, gas may leak away and the little pressure needed to move the droplet is not established in full, giving an artificially low reading of volume change. This was a challenge in all measurements, and great care was taken to obtain airtight fits. The airtightness was checked by applying about $1 \mathrm{kPa}$ of pressure, which is not sufficient to open the ET in humans (Sakakihara et al. 1993), and monitoring to check that pressure in the closed system was maintained.
A high-resolution device for automatic monitoring of gas volume changes $\left(\Delta V_{t}\right)$ with time $(t)$

The position of the liquid droplet inside the glass capillary was measured by using the custom-made optoelectronic device. A cold light source sends light into the back end of the horizontal capillary, which acts as an optical waveguide. The light is transmitted along the length of the capillary bore, and due to internal reflection almost no light is emitted in the perpendicular direction. Seen from the side, the capillary is practically dark. At the position of the droplet, light enters the fluid, and at the back and front end of the droplet, light is reflected in all directions by both menisci of the fluid. As the curvature of the proximal fluid meniscus is opposite to the propagation direction of the light, it reflects much more strongly than the distal meniscus. Hence, one sees the proximal meniscus as a tiny bright light spot in the capillary. Using mirrors and an imaging lens, the entire length of the capillary (about $19 \mathrm{~cm}$ ) is projected on a linear charge-coupled device (CCD) array. The capillary is covered so ambient light cannot reach the detector. Hence, all pixels are dark, except two to three pixels that are illuminated by the light reflected from the droplet. The CCD array delivers an analog output signal, where time corresponds to pixel position and voltage to pixel intensity. This signal is read about three times per second by a PC. Software determines the position of the intensity peak. In this way we obtain a position reading of the droplet meniscus about three times per second. The position data, together with the exact time when the position was determined, are stored in the computer memory.

As we used a 2048-pixel CCD array to measure $19 \mathrm{~cm}$ of capillary length, the theoretical resolution of the system is $0.092 \mathrm{~mm}$. The resolution of the position measurements depends also on the optics and the accuracy of the signal triggering, so we determined the actual resolution from a calibration measurement. This showed that the droplet position can actually be measured with accuracy better than $0.2 \mathrm{~mm}$. With a 0.72-mm-bore capillary, this corresponds to a volume measuring accuracy better than $0.08 \mu \mathrm{L}$.

\section{Data analysis: underlying assumptions and normalization methods}

The rate of transmucosal diffusion of gas from the blood perfusing the ME mucosa into the ME cavity was determined by using phase "a" data. This was done assuming that the rate-determining volume changes at the onset of the experiment are mostly due to the fast-diffusing $\mathrm{CO}_{2}$. 


\section{Normalization of phase "a" results}

The experimentally obtained curves of $\Delta V_{t}$ vs. $t$ were normalized to cancel effects such as the secretion of fluids from the surface of the ear canal or diffusion of other gases during phase "b," when observed.

Phase "b" was regarded as a constant shift in baseline. When both phases "a" and "b" were observed (e.g., Fig. 4), the phase "a" curve was normalized by using the corresponding values obtained from the extrapolation to $t=0$ of the linear equation fitted to phase "b." This was done assuming that the exchange (loss) of other gases in phase " $b$ " is linear with time (see the "Introduction").

In some cases, where a linear increase in volume was observed after phase "a" and fluid was found in the ear canal at the end of an experiment (e.g., Fig. 5), the linear increase in volume was attributed to continuous secretion of fluids into the closed volume of the ME system. Thus, experimental values were normalized by subtraction from the original exponential curve fitted to phase "a" [explained below; Eq. (2)], the corresponding values from the extrapolation to $t=0$ of the linear equation describing the increasing part of the curve.

In few cases, a plateau was observed from the end of phase "a" and after (e.g., Fig. 6). In these cases, phase "a" curves were not normalized.

Using calculations based on the volume change values obtained, the coefficient conductance of $\mathrm{CO}_{2}$ $\left(G \mathrm{CO}_{2}\right)$ for the mucosa was calculated as follows:

The diffusion rate of a gas through a permeable barrier in steady state can be described by Dejours (1981):

$$
\dot{V}_{\mathrm{g}}=\left(D_{\mathrm{g}} \times \alpha_{\mathrm{g}} \times \frac{A}{L}\right) \times \Delta P_{\mathrm{g}}
$$

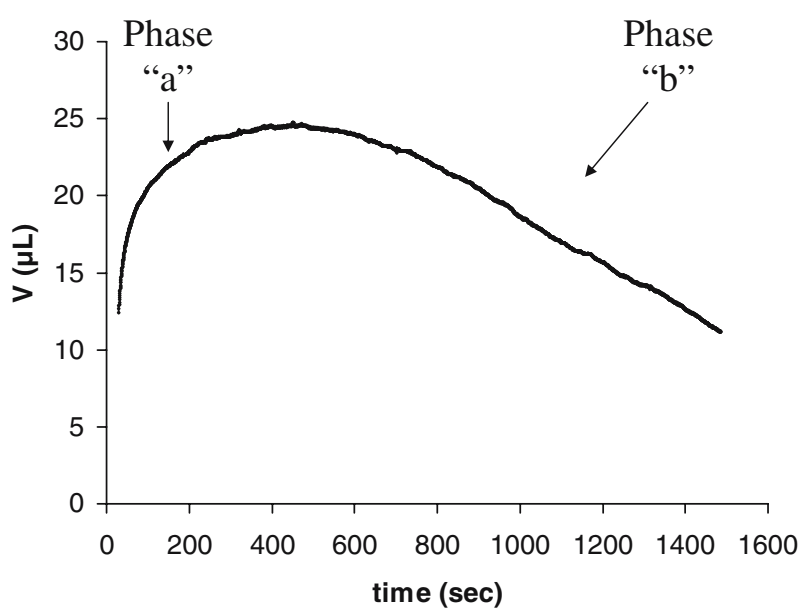

FIG. 4. A typical example of gas volume changes recorded (without pressure change) in the ME of a rabbit after flushing the ME with ambient air, demonstrating phases "a" and "b".

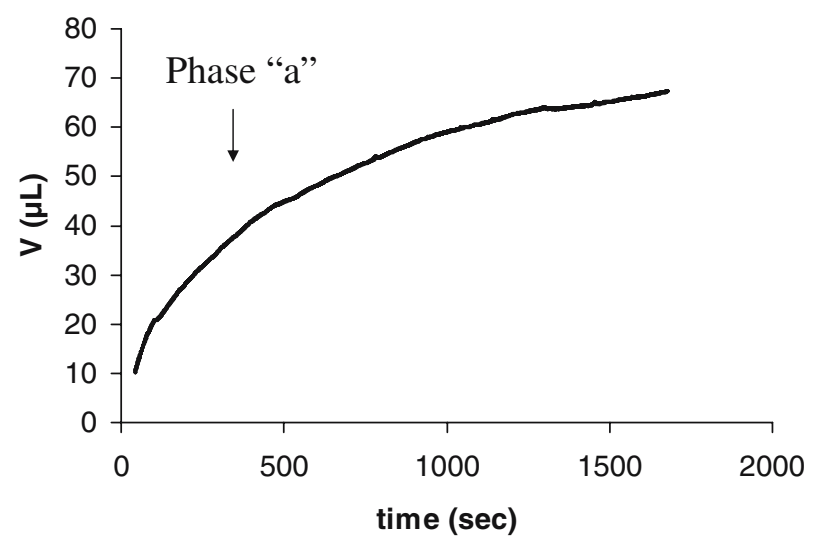

FIG. 5. An example of actual gas volume changes (phase "a") and further volume increase, possibly due to secretion of fluids into the closed system.

where $\dot{V}_{g}$ is the rate per unit of time of gas diffusion through the barrier thickness $L, D_{\mathrm{g}}$ is the diffusion coefficient of the gas in the barrier, $A$ is the surface area of the barrier, $\alpha_{\mathrm{g}}$ is the solubility coefficient of the gas in the barrier, and $\Delta P_{\mathrm{g}}$ is the partial pressure difference of a given gas across the barrier.

Or simply by lumping all physical and morphological constants of a given system into a gas conductance value, $G_{\mathrm{g}}$, we obtain $G_{\mathrm{g}}=\left(D_{\mathrm{g}} \times \alpha_{\mathrm{g}} \times \frac{A}{L}\right)$, and thus Eq. (1) becomes:

$$
\dot{V}_{g}=G_{\mathrm{g}} \times \Delta P_{\mathrm{g}}
$$

For $\mathrm{CO}_{2}, \mathrm{GCO}_{2}$ can thus be calculated using Eq. (1a) when $\dot{V} \mathrm{CO}_{2}$ and $\Delta \mathrm{PCO}_{2}$ are known.

\section{Calculation of $\Delta \mathrm{PCO}_{2}$}

The $\Delta \mathrm{PCO}_{2}$ across the ME mucosa was defined as the difference between the partial pressure of $\mathrm{CO}_{2}$ of the arterial blood $\left(\mathrm{PaCO}_{2}\right)$ perfusing the $\mathrm{ME}$ mucosa and the partial pressure of $\mathrm{CO}_{2}$ in the ME cavity.

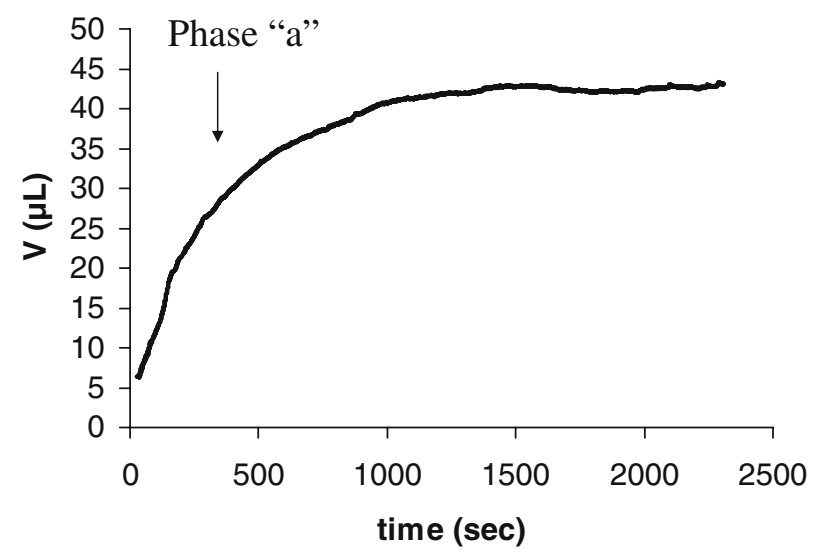

FIG. 6. An example of gas volume changes recording (phase "a") without further increase or decrease in volume. 
The approximate value of $\mathrm{PaCO}_{2}(\sim 6222 \mathrm{~Pa})$ during the time frame of the measurements was obtained from a previous study (Wyatt et al. 1989), where the New Zealand White rabbit was maintained under a similar protocol of anesthesia. This value was assumed to be similar in all the animals in the present study.

We assume that at the beginning of the experiment, after washing the ME with ambient air, the influence of $\mathrm{CO}_{2}$ production by the $\mathrm{ME}$ mucosa is negligible and the initial partial pressure of $\mathrm{CO}_{2}$ in the ME is very close to zero. Hence, at the beginning of the experiment, the value of $\Delta \mathrm{PCO}_{2} \cong \mathrm{PaCO}_{2}-\sim 0 \cong$ $\mathrm{PaCO}_{2}$.

We also assume that for very short periods of time Eq. (1) can describe $\dot{V} \mathrm{CO}_{2}$. Thus, initial $\dot{V} \mathrm{CO}_{2}$ at time zero $\left(\dot{V}_{\mathrm{i}} \mathrm{CO}_{2}\right)$, where other parameters of Eq. (1) are constant, is determined solely by $\mathrm{PaCO}_{2}$.

\section{Calculation of $\dot{V}_{\mathrm{i}} \mathrm{CO}_{2}$}

A first-order exponential curve was fitted to phase "a" in the form of:

$$
\Delta V_{t}=B\left(1-e^{-k t}\right)
$$

where $\Delta V_{t}$ is the deviation of ME volume starting from the closure time of the system, $k$ is the rate constant of gas volume increase, $t$ is elapsed time from system closure, and $B$ is the value of $\Delta V_{t}$ when $t$ approaches infinity.

It was assumed that the first derivative of the normalized Eq. (2) at $t=0, \Delta V_{t=0}=k B$, represents the initial $\dot{V} \mathrm{CO}_{2}$ from mucosal blood into the air-flushed ME cavity $\left(\dot{V}_{\mathrm{i}} \mathrm{CO}_{2}\right)$. It was obtained by fitting the normalized experimental data to Eq. (2) using the Simfit software (W.G. Bardsley, University of Manchester, UK). In cases where results were obtainẹd for both ears of the same rabbit, an animal average $V_{\mathrm{i}} \mathrm{CO}_{2}$ was used to overcome the dependence of results within the same animal.

\section{Calculation of $\mathrm{GCO}_{2}$}

Using the values of $\dot{V}_{\mathrm{i}} \mathrm{CO}_{2}$ from the present study, the $\Delta \mathrm{PCO}_{2}$ value of $6222 \mathrm{~Pa}$ obtained from a previous study (Wyatt et al. 1989), and using Eq. (1a), the values of $\mathrm{GCO}_{2}$ were calculated.

\section{RESULTS}

All three types of volume variations mentioned above were observed during the experiments:

1. Both phase "a" and phase "b" are obtained ( $n=9$; Fig. 4; Table 1). As can be seen in Fig. 4, the initial volume increase due to $\mathrm{CO}_{2}$ diffusing into the $\mathrm{ME}$

TABLE 1

\begin{tabular}{|c|c|c|c|}
\hline \multicolumn{4}{|c|}{ Initial $\mathrm{CO}_{2}$ influx into the $\mathrm{ME}\left(\dot{V}_{\mathrm{i}} \mathrm{CO}_{2}\right)$ : Results of normalization methods } \\
\hline Rabbit no. & Phase " $b$ " correction & "Fluids" correction & No correction \\
\hline R1 & 467.29 & & \\
\hline R2 & 159.88 & & \\
\hline R3 & 179.03 & & \\
\hline R4 & 329.67 & & \\
\hline R5 (2 ears) & & & $\begin{array}{l}\text { right, } 364.58 \\
\text { left, } 187.49 \\
\text { average, } 276.04\end{array}$ \\
\hline R6 (2 ears) & & $\begin{array}{l}\text { right, } 205.17 \\
\text { left, } 219.08 \\
\text { average, } 212.125\end{array}$ & \\
\hline R7 & & 445.42 & \\
\hline R8 & 282.97 & & \\
\hline R9 & 334.32 & & \\
\hline R10 & 298.08 & & \\
\hline R11 & & & 542.91 \\
\hline R12 & 495.57 & & \\
\hline R13 & 283.49 & & \\
\hline R14 & & 167.61 & \\
\hline R15 & & 322.23 & \\
\hline R16 & & 79.76 & \\
\hline Mean & 314 & 245 & 409 \\
\hline SD & \pm 112 & \pm 142 & \\
\hline$N$ & 9 & 5 & 2 \\
\hline
\end{tabular}

Values are in microliters per hour. See "Materials and methods" for details. 
lasts more than 500 s. After 800 s, a practically linear decrease is seen (phase " $b$ "), which has a much longer time scale than phase "a". Figure 4 demonstrates the extremely small volume changes that are involved in the process: The entire volume increase in phase "a" is less than $15 \mu \mathrm{L}$. Such extremely small values necessitate the use of a high-resolution device to obtain precise measurements (see "Materials and methods").

2. Phase "a" occurs, followed by a linear volume increase $(n=5$; Fig. 5; Table 1$)$. In all these five cases, fluid containing blood was found in the ear canal after the experiment. In our assumptions, we consider the rate of volume increase due to secretion of fluids to be constant throughout the measurements.

3. Initial volume increase (phase "a") followed by a plateau ( $n=2$; Fig. 6; Table 1$)$. In these cases, small amounts of fluid were found in the ear canal at the end of the experiments. Thus, apparently, the volume increase caused by secretion of fluids is cancelled by the volume decrease attributed to gas exchange.

In all the cases above, ears were checked for airtightness, and phase "a" was observed.

Table 1 presents the normalized results divided according to the three groups above. In two rabbits, results were obtained for both the left and right ears. Due to dependence within these animals, an average value was calculated for each of them.

The results of the three groups were not different (the large standard deviations around the means in these groups show that most of the results are within the same range of values). Thus, the overall mean initial rate of $\mathrm{CO}_{2}$ entrance into the $\mathrm{ME}$ was found to be $\dot{V}_{\mathrm{i}} \mathrm{CO}_{2}=305 \pm 131 \mu \mathrm{L} \cdot \mathrm{h}^{-1}($ mean $\pm \mathrm{SD}, n=16)$.

\section{DISCUSSION}

We have studied transmucosal $\mathrm{CO}_{2}$ exchange in the ME of the rabbit using an improved experimental setup based on the constant-pressure technique that was previously used in the rat (Kania et al. 2004). Automation of the measurement system in the present study, combined with the fact that the rabbit has a relatively large ME, enabled accurate recordings of small gas volume changes.

Data obtained from phase "a" (Figs. 4, 5, and 6) were used for the analysis under the previously supported assumption that the initial volume increase in that phase represents diffusion of $\mathrm{CO}_{2}$ from the blood into the air-flushed ME, down its partial pressures difference (Hamada et al. 2002).

To obtain as accurate a value of $\dot{V}_{\mathrm{i}} \mathrm{CO}_{2}$, as possible, we have corrected for the influence of other process- es that were presumably going on during the experiments, as explained in the "Materials and methods" section. Since the mean $\dot{V}_{\mathrm{i}} \mathrm{CO}_{2}$ was not significantly different between the three groups, an overall mean $\dot{V} \mathrm{CO}_{2}$ value of $305 \pm 131 \mu \mathrm{L} \cdot h^{-1}($ mean $\pm \mathrm{SD}, n=16)$ was calculated.

The apparent large standard deviation is rather typical for $\mathrm{ME}$ variables and in our case may be partially attributed to variability in ME mucosal blood flow, effective thickness, and partial gas pressures in the blood.

Using Eq. (1), we have evaluated the coefficient of conductance of $\mathrm{CO}_{2}\left(\mathrm{GCO}_{2}\right)$ of the ME mucosa of the rabbit, at an approximate temperature of $40^{\circ} \mathrm{C}$ [intermediate value between the body temperature of the rabbits as recorded in the present study and the body temperature reported by Wyatt et al. (1989)]: $G \mathrm{CO}_{2} \approx 0.05 \mu \mathrm{L}(\mathrm{h} \cdot \mathrm{Pa})^{-1}$ or $8.333 \times 10^{-4} \mu \mathrm{L} \cdot(\mathrm{min} \cdot \mathrm{Pa})^{-1}$. Because of the large standard deviation of the $\dot{V}_{\mathrm{i}} \mathrm{CO}_{2}$ used to calculate $\mathrm{GCO}_{2}$, and the possible (unmeasured) variation in $\mathrm{PaCO}_{2}$, we can only say that $\mathrm{GCO}_{2}$ of the rabbit ME mucosa must be anywhere between 0.5 and $1.2 \times 10^{-3} \mu \mathrm{L} \cdot(\min \cdot \mathrm{Pa})^{-1}$. Unfortunately, we have no literature data on rabbits to compare our results with. However, Fink et al. (2003) have estimated that $\mathrm{GCO}_{2}$ in humans is higher: $2.5 \times$ $10^{-10} \mathrm{~mol} \cdot(\mathrm{s} \cdot \mathrm{mmHg})^{-1}$, which is $\sim 0.34 \mu \mathrm{L}(\mathrm{min} \cdot \mathrm{Pa})^{-1}$ at $37^{\circ} \mathrm{C}$. When comparing the rabbit value to that of humans, one should take into account the other parameters that make up the conductance, in particular, the active surface area of the mucosa, which is not known yet for both species. However, assuming that diffusion distances are similar in all mammals, the $\mathrm{GCO}_{2}$ ratio may indicate that the active mucosal surface area of humans is two orders of magnitude higher than that of the rabbit.

\section{Influence of anesthesia}

ET activity. We have assumed that because the rabbits were maintained under general anesthesia during the experiments, the ET did not open spontaneously and no ventilation occurred via the ET. This assumption was proved experimentally to be true in rats (Kania et al. 2004), and the similarity in the pattern of the curves between their study and ours provides further support to this assumption. In both studies, the curves obtained were rather "smooth," in contrast to fast up/down pressure changes observed in awake humans (Mover-Lev et al. 1998; Tideholm et al. 1998), which could be attributed to deglutition events.

Influence on the arterial $\mathrm{PaCO}_{2}$. It has been shown in New Zealand White rabbits by Wyatt et al. (1989) that intramuscular induction of anesthesia with ketamine and xylazine followed by constant infusion of the 
anesthetic mix leads to an increase in arterial $\mathrm{PaCO}_{2}$. In their study, the mean $\mathrm{PaCO}_{2}$ increased to $\approx 46.67$ $\mathrm{mmHg}(\approx 6222 \mathrm{~Pa})$ within $30 \mathrm{~min}$ after initiation of the infusion (estimated from Fig. 1). $\mathrm{PaCO}_{2}$ stayed approximately constant for 120 minutes after initiation of the infusion followed by a further increase up to a maximum of $50.2 \mathrm{mmHg}(\approx 6693 \mathrm{~Pa} ; 66.5 \%$ above baseline) after $3 \mathrm{~h}$ of infusion. However, for our purposes, such a change, which presumably stems from reduced ventilation, is not manifested in our experiments since we used early data (phase "a") for our analysis.

\section{CONCLUSIONS}

An experimental setup has been developed that allows studying small gas volume changes in an animal's ME, with both high volume resolution $(0.08 \mu \mathrm{L})$ and high time resolution $(3 \mathrm{~Hz})$. Using the initial volume changes after washing the ME with air and assuming that it represents mainly $\mathrm{CO}_{2}$ penetration, we have studied the $\mathrm{ME}$ transmucosal $\mathrm{CO}_{2}$ exchange in the rabbit. An initial flow rate of $305 \pm 131 \mu \mathrm{L} \cdot \mathrm{h}^{-1}$ (mean $\pm \mathrm{SD}, n=16$ ) was found. Compared to the rabbit ME volume of ca. $300 \mu \mathrm{L}$, this value demonstrates that mucosal gas exchange and gas conductance are major factors in ME gas economy.

\section{ACKNOWLEDGMENTS}

This study was supported by a grant from the University of Antwerp (NOI project no. BOF/44750) and by grants from the Fund for Scientific Research (FWO). One of us (A.A.) was partially supported by the Nicholas and Elizabeth Shlezak Super Center for Cardiology and Biomedical Engineering, Tel Aviv University (No. 940110). We thank Fred Wiese and William Deblauwe for their technical assistance and Ann Belinsky for reading the manuscript and for her valuable comments.

\section{REFERENCES}

Aoki K, Mitani Y, Tuji T, Hamada Y, Utahashi H, Moriyama H. Relationship between middle ear pressure, mucosal lesion, and mastoid pneumatization. Laryngoscope 108:1840-1845, 1998.

ar a, Marcusohn Y, Sadé J, Kania R, Lecain E, Herman P, Tran Ba Huy $P$. The middle ear as a 'small lung' with a low ventilationto-perfusion ratio: a quantitative model for its gas exchange, effective blood flow and mucosa thickness in the rat. IXth Oxford conference on modeling and control of breathing. Postgenomic perspectives in modeling and control of breathing, Paris, September 2003.

Dejours P. Principles of comparative respiratory physiology, 2nd ed. Elsevier/North-Holland Biomedical Press, Amsterdam 1981.

Dirckx JJJ, Somers T, Decraemer WF, Govaerts P, Offeciers E.
Continuous pressure monitoring in the intact middle ear. In: Magnan J, Chays A (eds) Cholesteatoma and ear surgery, pp 41-47, 2001.

Doyle WJ, Alper CM. A model to explain the rapid pressure decrease after air-inflation of diseased middle ears. Laryngoscope 109:70-78, 1999.

Doyle WJ, Seroky JT. Middle ear gas exchange in rhesus monkeys. Ann. Otol. Rhinol. Laryngol. 103:636-645, 1994.

Doyle WJ, Seroky JT, Alper CM. Gas exchange across the middle ear mucosa in monkeys. Estimation of exchange rate. Arch. Otolaryngol. Head Neck Surg. 121:887-892, 1995.

Doyle WJ, Alper CM, Seroky JT. Trans-mucosal inert gas exchange constants for the monkey middle ear. Auris Nasus Larynx 26:5$12,1999$.

Eden AR, Gannon PJ. Neural control of middle ear aeration. Arch. Otolaryngol. Head Neck Surg. 113:133-137, 1987.

Eden AR, Laitman JT, Gannon PJ. Mechanisms of middle ear aeration: anatomic and physiologic evidence in primates. Laryngoscope 100:67-75, 1990.

FeLDING JU. Middle ear gas-its composition in the normal and in the tubulated ear. A methodological and clinical study. Acta Otolaryngol. Suppl. 536:1-57, 1998.

Fink N, Ar A, SADÉ J, BARNEA O. Mathematical analysis of atelectasis formation in middle ears with sealed ventilation tubes. Acta Physiol. Scand. 177:493-505, 2003.

Goksu N, Haziroglu R, Kemaloglu Y, Karademir N, Bayramoglu I, AKYıldiz N. Anatomy of the guinea pig temporal bone. Ann. Otol. Rhinol. Laryngol. 101:699-704, 1992.

Hamada Y, Utahashi H, Aoki K. Physiological gas exchange in the middle ear cavity. Int. J. Pediatr. Otorhinolaryngol. 64:41-49, 2002.

Hellström S, Stenfors LE. The pressure equilibrating function of pars flaccida in middle ear mechanics. Acta Physiol. Scand. 118:337-341, 1983.

Hellström S, Salén B, Stenfors LE. Anatomy of the rat middle ear. A study under the dissection microscope. Acta Anat. 112:346352, 1982.

Judkins RF, Li H. Surgical anatomy of the rat middle ear. Otolaryngol. Head Neck Surg. 117:438-447, 1997.

Kania R, Portier F, Lecain E, Marcusohn Y, Ar A, Herman P, Tran BA HuY P. Experimental model for investigating trans-mucosal gas exchanges in the middle ear of the rat. Acta Otolaryngol. 124:408-410, 2004.

Kanick SC, Doyle WJ, Ghadiali SN, Federspiel WJ. On morphometric measurement of oxygen diffusing capacity in middle ear gas exchange. J. Appl. Physiol. 98:114-119, 2005.

Mover-Lev H, Priner-Barenholtz R, Ar A, Sadé J. Quantitative analysis of gas losses and gains in the middle ear. Respir. Physiol. 114:143-151, 1998.

NagaI T. Innervation of the tympanic membrane. Acta Otorhinolaryngol. Belg. 49:117-120, 1995.

Nagai T, Tono T. Encapsulated nerve corpuscles in the human tympanic membrane. Arch. Otorhinolaryngol. 246:169-172, 1989.

Nielsen LG, Sami AKS, Drewes AM, Nielsen KD, Gaihede M. Central control of human middle ear pressure: preliminary evidence based on brain evoked potentials. Otol Neurotol 26(2):305306. In: Dirckx JJJ, Sadé J. Middle ear pressure regulation: basic research and clinical observation. Otol Neurotol 26(2): 305-309, 2005.

Pracy JP, White A, Mustafa Y, Smith D, Perry ME. The comparative anatomy of the pig middle ear cavity: a model for middle ear inflammation in the human? J. Anat. 192:359-368, 1998.

SADÉ J, AR A. Middle ear and auditory tube: middle ear clearance, gas exchange and pressure regulation. Otolaryngol. Head Neck Surg. 116:499-524, 1997.

SADÉ J, Ar A, Marcusohn Y. The hyperectatic outwardly ballooned 
tympanic membrane as an indicator of an active mechanism of middle ear aeration. Otol. Neurotol. 26(2):306-307. In: Dirckx JJJ, Sadé J. Middle ear pressure regulation: basic research and clinical observation. Otol Neurotol 26(2):305-309, 2005.

Sakakihara J, Honjo I, Fujita A, Kurata K, Takahashi H. Compliance of the patulous eustachian tube. Ann. Otol. Rhinol. Laryngol. 102:110-112, 1993.

Tideholm B, Carlborg B, Jönsson S, Bylander-Groth A. Continuous long-term measurements of the middle ear pressure in subjects without a history of ear disease. Acta Otolaryngol. (Stockh) 118:369-374, 1998.
Uchimizu H, Utahashi H, Hamada Y, Aoki K. Middle ear total pressure measurement as a useful parameter for outcome prediction in pediatric otitis media with effusion. Int. J. Pediatr. Otorhinolaryngol. 69(12):1659-1665, 2005.

WILSON JG. The nerves and nerve endings in the membrana tympani of man. Am. J. Anat. 11(2):101-112, 1911.

Wyatt JD, Scott RA, Richardson ME. The effects of prolonged ketamine-xylazine intravenous infusion on arterial blood $\mathrm{pH}$, blood gases, mean arterial blood pressure, heart and respiratory rates, rectal temperature and reflexes in the rabbit. Lab. Anim. Sci. 39:411-416, 1989. 